\title{
Sound propagation over a ground with irregularity
}

\author{
Yasushi Miki \\ Institute of Interdisciplinary Research, \\ Faculty of Engineering, University of Tokyo, \\ 6-1 Komaba 4-chome, Meguro-ku, Tokyo, 153 Japan
}

(Received 17 September 1981)

\begin{abstract}
A theory of sound propagation based on Niessen's method is developed. The velocity potential at a given point above a boundary is expressed in the form of surface integral on the boundary. The advantage of the theory is that it is applicable not only to a homogeneous plane surface but also to a surface with irregularity or a composite surface of different kinds. Theoretical examinations are made in three cases, i.e., a boundary of perfect reflection, a boundary of finite impedance and a boundary of finite impedance with irregularity. In the last examination, it is assumed that the boundary is almost flat but has some concaved portions distributed with a certain pattern. On this assumption, the theoretical result is found in good agreement with the experimental result measured over a turf-covered ground. A way of applying the theory to the case of a composite surface is also described. In connection with the theory, the strict mathematical formulation of Huygens principle is given.
\end{abstract}

PACS number: 43. 20. Bi, 43. 28. Fp

\section{INTRODUCTION}

It is well known that theories of sound propagation by Rudnick ${ }^{1)}$ and Ingard $^{2)}$ are based on the theories of electromagnetic wave propagation by Sommerfeld and Weyl, ${ }^{3)}$ respectively. In these theories the ground surface is assumed to be a homogeneous plane boundary. From a practical point of view, however, this assumption does not necessarily hold since a composite surface of different kinds or a surface with irregularity is usually the case. In this paper, a theory of sound propagation based on Niessen's method ${ }^{4}$ is developed. The velocity potential at a given point above a boundary is expressed in the form of surface integral on the boundary. Accordingly the theory is applicable to the case of a composite surface and, on some assumptions, to the case of a rough surface. ${ }^{5}$ )

As described in a previous paper, ${ }^{6)}$ irregular responses seen in the experimental results over a turf-covered ground are considered to be the effects of ground irregularities. In order to see how the ground irregularities affect the propagation characteristics, we first estimate the irregularity pattern of the surface from the experimental data assuming that the surface is almost flat but has some concaved portions. Then a theoretical examination is made and shown to be in good agreement with the experimental result.

\section{REPRESENTATION OF NIESSEN'S THEORY IN ACOUSTIC SYSTEM}

We consider the velocity potential at point $\mathrm{R}$ due to a point source $\mathrm{S}$ above a plane surface $f_{1}$ shown in Fig. 1. Let $\Psi$ and $u$ be two independent solutions of Helmholtz Eq.

$$
\nabla^{2} \Psi+k_{1}^{2} \Psi=0,
$$

and apply Green's theorem to the shadowless domain which is bounded by the surface $f_{1}$ and infinitely small spheres $f_{2}, f_{3}$ with center at $\mathrm{R}, \mathrm{S}$. Then $\Psi$ is given as the solution of

$$
\int_{f_{1}+f_{2}+f_{3}}\left(u \frac{\partial \Psi}{\partial n}-\Psi \frac{\partial u}{\partial n}\right) d f=0
$$




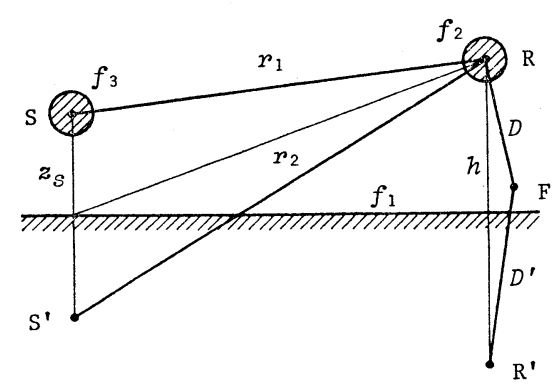

Fig. 1 Geometry of source and receiver.

where $d f$ is a surface element and $n$ the outer normal. If we choose

$$
u=\frac{\exp \left[i k_{1} D\right]}{D}+\frac{\exp \left[i k_{1} D^{\prime}\right]}{D^{\prime}},
$$

where $D$ and $D^{\prime}$ are the distances from a variable point $F$ to $R$ and $R^{\prime}$ (image point of $R$ ), respectively, the integral of Eq. (2.2) for each surface $f_{1}, f_{2}, f_{3}$ becomes

$$
\begin{aligned}
& f_{1}: \int\left(u \frac{\partial \Psi}{\partial n}-\Psi \frac{\partial u}{\partial n}\right) d f_{1} \\
&=-2 \int \Psi_{f_{1}} \frac{\partial}{\partial n}\left(\frac{\exp \left[i k_{1} D\right]}{D}\right) d f_{1}, \\
& f_{2}: \int\left(u \frac{\partial \Psi}{\partial n}-\Psi \frac{\partial u}{\partial n}\right) d f_{2}=-4 \pi \Psi_{\mathrm{R}}, \\
& f_{3}: \quad \int\left(u \frac{\partial \Psi}{\partial n}-\Psi \frac{\partial u}{\partial n}\right) d f_{3} \\
&=4 \pi\left(\frac{\exp \left[i k_{1} r_{1}\right]}{r_{1}}-\frac{\exp \left[i k_{1} r_{2}\right]}{r_{2}}\right) .
\end{aligned}
$$

Then, $\Psi_{\mathrm{R}}$, the velocity potential at point $\mathrm{R}$, is given by

$$
\begin{aligned}
\Psi_{\mathrm{R}}= & \frac{\exp \left[i k_{1} r_{1}\right]}{r_{1}}-\frac{\exp \left[i k_{1} r_{2}\right]}{r_{2}} \\
& -\frac{1}{2 \pi} \int \Psi_{f_{1}} \frac{\partial}{\partial n}\left(\frac{\exp \left[i k_{1} D\right]}{D}\right) d f_{1},
\end{aligned}
$$

where $\Psi_{f_{1}}$ is the velocity potential on the surface $f_{1}$, and according to Rudnick (Eqs. (15), (16) in Ref. 1)),

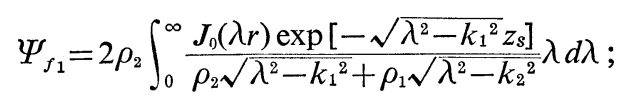

where $J_{0}(\cdot)$ is the Bessel function of order zero; $k_{1}\left(k_{2}\right)$ and $\rho_{1}\left(\rho_{2}\right)$ are the wave number and the density of upper (lower) medium, respectively; $r$ is the horizontal distance between the point $\mathbf{S}$ and a given point on the surface $f_{1}$; and $z_{s}$ the height of $\mathbf{S}$.

In the special case that $z_{s}=0$, Eq. (2.7) is expressed with suffix 0 as

$$
\Psi_{\mathrm{R}}^{0}=-\frac{1}{2 \pi} \int \Psi_{f_{1}^{0}}^{0} \frac{\partial}{\partial n}\left(\frac{\exp \left[i k_{1} D\right]}{D}\right) d f_{1},
$$

and Eq. (2.8) as

$$
\begin{gathered}
\Psi_{f_{1}}^{0}=2 A \int_{\xi=1}^{k_{2} / k_{1}} \frac{\exp \left[i k_{1} r \xi\right]}{r} d\left(\frac{s}{\sqrt{\xi^{2}-s^{2}}}\right), \\
A=\frac{\sqrt{k_{1}{ }^{2}-k_{2}{ }^{2}}}{\sqrt{\rho_{1}{ }^{2} k_{2}{ }^{2}-\rho_{2}{ }^{2} k_{1}{ }^{2}}} \frac{\rho_{1} \rho_{2}{ }^{2}}{\rho_{1}{ }^{2}-\rho_{2}{ }^{2}}, \\
s^{2}=\frac{\rho_{1}{ }^{2} k_{2}{ }^{2}-\rho_{2}{ }^{2} k_{1}{ }^{2}}{k_{1}{ }^{2}\left(\rho_{1}{ }^{2}-\rho_{2}{ }^{2}\right)},
\end{gathered}
$$

by the use of identities

$$
\begin{aligned}
& \int_{\xi=1}^{k_{2} / k_{1}} \frac{\lambda}{\sqrt{\lambda^{2}-k_{1}^{2} \xi^{2}}} d\left(\frac{s}{\sqrt{\xi^{2}-s^{2}}}\right) \\
& =\frac{1}{A} \frac{\lambda}{\rho_{2} \sqrt{\lambda^{2}-k_{1}^{2}}+\rho_{1} \sqrt{\lambda^{2}-k_{2}^{2}}}, \\
& \frac{\exp \left[i k_{1} r \xi\right]}{r}=\int_{0}^{\infty} \frac{J_{0}(\lambda r)}{\sqrt{\lambda^{2}-k_{1}{ }^{2} \xi^{2}}} \lambda d \lambda .
\end{aligned}
$$

\section{THE NEW THEORY}

3.1 Expressions for a Surface of Local Reaction

In Sec. 2, the boundary is considered to be one of extended reaction. ${ }^{7)}$ In the case of local reaction, as is generally assumed for a ground surface, the same arguments are valid with additional requirements

$$
\frac{\rho_{1} k_{2}}{\rho_{2} k_{1}}=v, \quad\left|\frac{\rho_{2}}{\rho_{1}}\right| \rightarrow \infty, \quad\left|\frac{k_{2}}{k_{1}}\right| \rightarrow \infty,
$$

where $\nu$ is the specific admittance. Then Eqs. (2.11) and (2.12) are simplified to

$$
\begin{gathered}
A=\frac{-v}{\sqrt{1-\nu^{2}}}, \\
s^{2}=1-\nu^{2},
\end{gathered}
$$

and Eq. (2.10) is expressed as

$$
\Psi_{f_{1}}^{0}=-\frac{\nu}{\sqrt{1-\nu^{2}}} \int_{\xi=1}^{\infty} \frac{\exp [i k r \xi]}{r} d\left(\frac{s}{\sqrt{\xi^{2}-s^{2}}}\right),
$$

(henceforth the symbol $k$ is used instead of $k_{1}$ ). Substituting Eq. (3.4) into Eq. (2.9), we obtain

$$
\begin{aligned}
\Psi_{R}^{0}= & \frac{\nu}{\pi \sqrt{1-\nu^{2}}} \int_{\xi=1}^{\infty} \int_{0}^{2 \pi} \int_{0}^{\infty} \exp [i k(r \xi+D)] \\
& \times\left(i k-\frac{1}{D}\right) \frac{h}{D^{2}} d r d \phi d\left(\frac{s}{\sqrt{\xi^{2}-s^{2}}}\right),
\end{aligned}
$$




\section{Y. MIKI: SOUND PROPAGATION OVER GROUND}

where following relations are used:

$$
\begin{aligned}
d f_{1} & =r d r d \phi \\
\frac{\partial}{\partial n}\left(\frac{\exp [i k D]}{D}\right) & =\left(i k-\frac{1}{D}\right) \frac{h}{D} \frac{\exp [i k D]}{D} .
\end{aligned}
$$

3.2 Identity of Solutions by Rudnick and by Ingard

We consider the special case that both source and receiver are on the boundary of local reaction. Equation (3.4) is a solution derived from the solution by Rudnick (Eq. (2.8)), and can be expressed in the form

$$
\Psi_{f_{1}}^{0}=2 i k \int_{1}^{\infty} \exp [i k r \xi]\left(\frac{v}{\sqrt{\xi^{2}-s^{2}}}-1\right) d \xi .
$$

According to Ingard (Eqs. (2) (6) in Ref. 2)),

$$
\Psi_{f_{1}}^{0}=-i k \int_{1}^{i \infty} \exp [i k r \gamma]\left(1+\left\langle R_{p}(\theta)\right\rangle_{\mathrm{av}}\right) d \gamma,
$$

where, in this case, $\theta=\pi / 2$, and with $\gamma=\cos \eta$,

$$
\begin{aligned}
\left\langle R_{p}(\pi / 2)\right\rangle_{\mathrm{av}} & =\frac{1}{2 \pi} \int_{0}^{2 \pi} \frac{\sin \eta \cos \psi-\nu}{\sin \eta \cos \psi+\nu} d \psi \\
& =1-\frac{2 \nu}{\sqrt{\nu^{2}-1+\gamma^{2}}} \\
& =1-\frac{2 \nu}{\sqrt{\gamma^{2}-s^{2}}} .
\end{aligned}
$$

Then Eq. (3.9) becomes

$$
\Psi_{f_{1}^{0}}^{0}=2 i k \int_{1}^{i \infty} \exp [i k r \gamma]\left(\frac{v}{\sqrt{\gamma^{2}-s^{2}}}-1\right) d \gamma .
$$

Since the upper limits of integrations in Eqs. (3.8) and (3.11) mean $|\xi| \rightarrow \infty$ and $\operatorname{Im}[\gamma] \rightarrow \infty$, respectively, the integration path of the former can be replaced by that of the latter. Then two equations, i.e., the solutions by Rudnick and by Ingard become identical.

\subsection{General Expressions}

The most important result of Niessen's theory is that the velocity potential at point $\mathrm{R}$ is expressed in the form of surface integral on the boundary $f_{1}$, i.e., from Eqs. (2.7) and (3.7),

$$
\begin{aligned}
\Psi_{R}= & \frac{\exp \left[i k r_{1}\right]}{r_{1}}-\frac{\exp \left[i k r_{2}\right]}{r_{2}} \\
& -\frac{1}{2 \pi} \int \Psi_{f_{1}}\left(i k-\frac{1}{D}\right) \frac{h}{D^{2}} \exp [i k D] d f_{1} .
\end{aligned}
$$

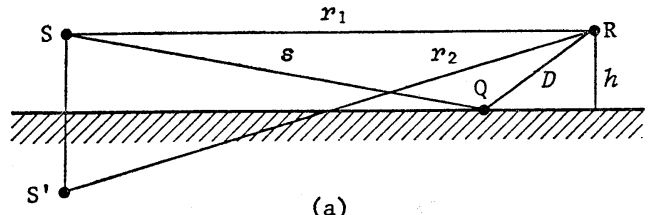

(a)

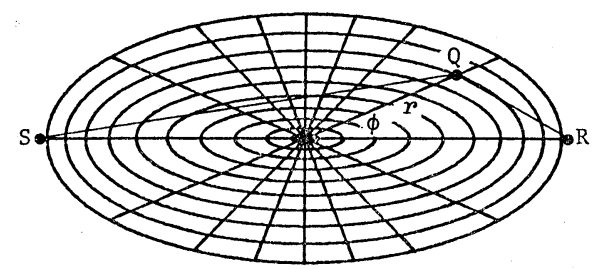

(b)

Fig. 2 (a) Horizontal arrangement of source and receiver above a flat surface.

(b) Top view of the surface. Ellipses (Eq. (3.23)) represent the "equi-propagation-time" curves.

In the acoustic system shown in Fig. 2, let $\mathrm{Q}(r, \phi)$ be a point on the boundary. Then

$$
\begin{aligned}
\Psi_{R}= & \frac{\exp \left[i k r_{1}\right]}{r_{1}}-\frac{\exp \left[i k r_{2}\right]}{r_{2}} \\
& -\frac{1}{2 \pi} \int_{0}^{2 \pi} \int_{0}^{\infty} \Psi_{Q}\left(i k-\frac{1}{D}\right) \frac{h}{D^{2}} \\
& \cdot \exp [i k D] r d r d \phi, \\
\Psi_{Q} & =\frac{\exp [i k s]}{s}+\Psi_{\mathrm{Q}}{ }^{\prime},
\end{aligned}
$$

where $\Psi_{\mathrm{Q}}$ is the velocity potential at point $\mathrm{Q}$ and $\Psi_{\mathrm{Q}}{ }^{\prime}$ is the contribution from image source $\mathrm{S}^{\prime}$. Substituting Eq. (3.14) into Eq. (3.13) and using the identity (which will be proved in Sec. 3.4)

$$
\begin{array}{r}
-\frac{1}{2 \pi} \int_{0}^{2 \pi} \int_{0}^{\infty} \frac{\exp [i k s]}{s}\left(i k-\frac{1}{D}\right) \frac{h}{D^{2}} \\
\cdot \exp [i k D] r d r d \phi=\frac{\exp \left[i k r_{2}\right]}{r_{2}},
\end{array}
$$

we obtain

$$
\begin{aligned}
\Psi_{\mathrm{R}}= & \frac{\exp \left[i k r_{1}\right]}{r_{1}} \\
& -\frac{1}{2 \pi} \int_{0}^{2 \pi} \int_{0}^{\infty} \Psi_{\mathrm{Q}}^{\prime}\left(i k-\frac{1}{D}\right) \frac{h}{D^{2}} \\
& \cdot \exp [i k D] r d r d \phi .
\end{aligned}
$$

In Eq. (3.16), the first term represents the contribution from source $S$, and the second term from image 
source $\mathrm{S}^{\prime}$.

Next, let $a$ be the length of path SQR. Then

$$
\begin{aligned}
a & =s+D, \\
s^{2} & =\left(x+\frac{r_{1}}{2}\right)^{2}+y^{2}+h^{2}, \\
D^{2} & =\left(x-\frac{r_{1}}{2}\right)^{2}+y^{2}+h^{2}, \\
x & =r \cos \phi, \\
y & =r \sin \phi, \\
r_{2}{ }^{2} & =r_{1}{ }^{2}+4 h^{2},
\end{aligned}
$$

and we get

$$
r^{2}=\frac{a^{2}\left(a^{2}-r_{2}^{2}\right)}{4\left(a^{2}-r_{1}^{2} \cos ^{2} \phi\right)} .
$$

Equation (3.23) shows a locus of point $\mathrm{Q}$ on the boundary, and it describes an ellipse when $a$ is constant. The ellipses corresponding to several values of $a$ are shown in Fig. 2. These are, in acoustical sense, "equi-propagation-time" curves, and the reflected waves from points on the same ellipse reach the receiver at the same time.

From Eq. (3.23),

$$
r \frac{d r}{d a}=\frac{a\left(a^{4}-2 a^{2} r_{1}^{2} \cos ^{2} \phi+r_{1}^{2} r_{2}^{2} \cos ^{2} \phi\right)}{4\left(a^{2}-r_{1}^{2} \cos ^{2} \phi\right)^{2}},
$$

and finally we obtain

$$
\begin{aligned}
\Psi_{\mathrm{R}}= & \frac{\exp \left[i k r_{1}\right]}{r_{1}} \\
& -\frac{1}{2 \pi} \int_{0}^{2 \pi} \int_{r_{2}}^{\infty} \Psi_{\mathrm{Q}}^{\prime}\left(i k-\frac{1}{D}\right) \frac{h}{D^{2}} \\
& \cdot \exp [i k D]\left(r \frac{d r}{d a}\right) d a d \phi .
\end{aligned}
$$

3.4 Mathematical Formulation of Huygens Principle

In this section, we shall first prove the identity indicated by Eq. (3.15) in Sec. 3.3, and then discuss its physical meaning.

[Proof of Eq. (3.15)]

Let $\mathrm{S}^{\prime}$ and $\mathrm{R}$ be arbitrary points in the free space and $f_{1}$ be the plane which intersects with the segment $\mathrm{S}^{\prime} \mathrm{R}$ at 0 , the middle point of the segment, as shown in Fig. 3. Then the left-hand side of Eq. (3.15), denoted by $\Psi$, becomes

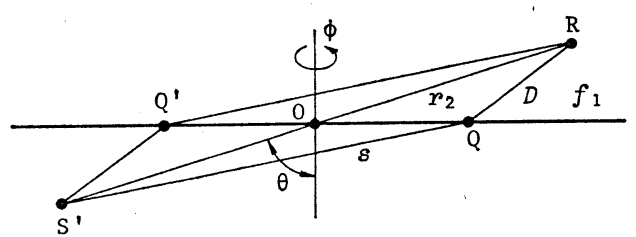

Fig. 3 Illustration for deriving the mathematical formulation of Huygens principle (Eq. (3.15)).

$$
\begin{aligned}
\Psi= & -\frac{1}{2 \pi} \int_{0}^{2 \pi} \int_{0}^{\infty} \frac{\exp [i k s]}{s}\left(\frac{i k}{D}-\frac{1}{D^{2}}\right) \\
& \cdot \frac{r_{2} \cos \theta}{2} \frac{\exp [i k D]}{D} r d r d \phi \\
s^{2} & =r^{2}+r r_{2} \sin \theta \cos \phi+\frac{1}{4} r_{2}{ }^{2}, \\
D^{2} & =r^{2}-r r_{2} \sin \theta \cos \phi+\frac{1}{4} r_{2}{ }^{2}
\end{aligned}
$$

Taking the symmetry of $\mathrm{Q}$ and $\mathrm{Q}^{\prime}$ into account, we have

$$
\begin{aligned}
\Psi= & -\frac{1}{2 \pi} \int_{0}^{\pi} \int_{0}^{\infty} \exp [i k(s+D)]\left\{i k\left(\frac{1}{s}+\frac{1}{D}\right)\right. \\
& \left.-\left(\frac{1}{s^{2}}+\frac{1}{D^{2}}\right)\right\} \frac{r_{2} \cos \theta}{2 s D} r d r d \phi .
\end{aligned}
$$

Since the integrand can be expressed in the total differential form

$$
\begin{aligned}
\exp & {[i k(s+D)]\left\{i k\left(\frac{1}{s}+\frac{1}{D}\right)-\left(\frac{1}{s^{2}}+\frac{1}{D^{2}}\right)\right\} \frac{r}{s D} } \\
= & \frac{d}{d r}\left\{\left(\frac{1}{s}+\frac{1}{D}\right) \frac{s+D}{(s+D)^{2}-\left(r_{2} \sin \theta \cos \phi\right)^{2}}\right. \\
& \cdot \exp [i k(s+D)]\},
\end{aligned}
$$

and $s=D=r_{2} / 2$ at $r=0$, we obtain

$$
\begin{aligned}
\Psi & =\frac{\exp \left[i k r_{2}\right]}{r_{2}} \frac{\cos \theta}{\pi} \int_{0}^{\pi} \frac{1}{1-(\sin \theta \cos \phi)^{2}} d \phi \\
& =\frac{\exp \left[i k r_{2}\right]}{r_{2}} .
\end{aligned}
$$

\section{Q.E.D.}

It is obvious that Eq. (3.31) is the velocity potential at point $R$ due to the point source at $S^{\prime}$. Using the velocity potential at $Q$, i.e.,

$$
\Psi_{\mathrm{Q}}=\frac{\exp [i k s]}{s},
$$


we can express Eq. (3.15) as

$$
\begin{gathered}
\Psi=\int_{0}^{2 \pi} \int_{0}^{\infty} \Psi_{Q} \frac{\exp [i k D]}{D} K(\mathcal{X}) r d r d \phi, \\
K(\chi)=-\frac{1}{2 \pi}\left(i k-\frac{1}{D}\right) \frac{h}{D} \\
=-\frac{1}{2 \pi}\left(i k-\frac{1}{D}\right) \cos \chi,
\end{gathered}
$$

where $\mathcal{X}$ is the angle between the normal at $\mathrm{Q}$ and the segment $\mathrm{QR}$, and $\cos \lambda=h / D$. Equation (3.33) is interpreted as follows:

The source $\mathbf{S}^{\prime}$ generates an infinite number of secondary sources on a given plane, each of which has the magnitude $\Psi_{\mathrm{Q}}$ and the directivity $K(\chi)$, and the velocity potential at point $\mathrm{R}$ is given by the summation of the contributions from them.

Thus, Eqs. (3.33) and (3.34) are the strict mathematical formulation of Huygens principle. In the case where

$$
s, D \gg 1 / k,
$$

the approximate directivity becomes

$$
K(\chi) \fallingdotseq-\frac{i k}{2 \pi} \cos \chi,
$$

which is similar but not identical to Kirchhoff's approximation $^{8)}$

$$
K(\chi) \fallingdotseq-\frac{i k}{4 \pi}(1+\cos \chi)
$$

\section{CALCULATIONS OF SOUND PROPAGATION CHARACTERISTICS BY THE NEW THEORY}

In the following sections, we offer two examples of theoretical calculations of sound propagation over some practical boundaries.

\subsection{Boundary of Perfect Reflection}

We pay attention to reflection characteristics and consider only the second term in Eq. (3.25):

$$
\begin{aligned}
\Psi_{\mathrm{R}}^{\prime}= & -\frac{1}{2 \pi} \iint \Psi_{\mathrm{Q}}^{\prime}\left(i k-\frac{1}{D}\right) \frac{h}{D^{2}} \exp [i k D] \\
& \cdot\left(r \frac{d r}{d a}\right) d a d \phi,
\end{aligned}
$$

where, in this case,

$$
\Psi_{\mathrm{Q}}{ }^{\prime}=\frac{\exp [i k s]}{s} .
$$

Then Eq. (4.1) must be

$$
\Psi_{\mathrm{R}}{ }^{\prime}=\frac{\exp \left[i k r_{2}\right]}{r_{2}},
$$

by Eq. (3.15). Therefore, we can check the accuracy of numerical integration of Eq. (4.1) by comparing the result with Eq. (4.3).

Variables of integration are

$$
\begin{gathered}
a=r_{2}+\left\{\frac{1}{50}\left(m-\frac{1}{2}\right)\right\}^{3}, \quad m=1,2, \cdots, 50, \\
\phi=\frac{\pi}{80}\left(n-\frac{1}{2}\right), \quad n=1,2, \cdots, 80,
\end{gathered}
$$

and the horizontal distance $r_{1}$ and the height $h$ are chosen to be

$$
\begin{aligned}
& r_{1}=10 \mathrm{~m}, \\
& h=140,73.3,33.3,13.3 \mathrm{~cm},
\end{aligned}
$$

as in Ref. 6). For $h=140 \mathrm{~cm}$, the area of integration is shown in Fig. 2.

The results are given in the forms of triangular

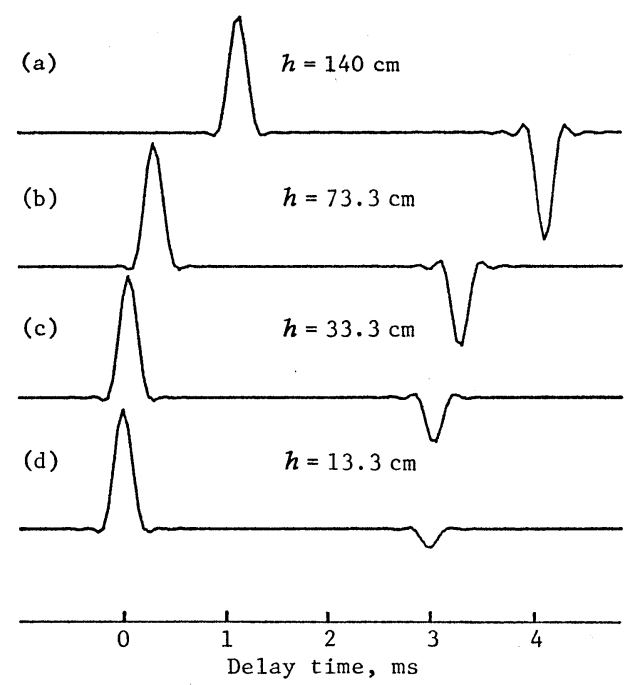

Fig. 4 Reflected wave components of triangular impulse from the boundary of perfect reflection with the arrangement of Fig. 2, calculated from Eqs. (4.1), (4.2) and (4.4) (4.7).

The component in the latter half of each response is the error caused by the finite upper limit of integration for $a$ (Eq. 4.4)). The horizontal axis is the relative delay time from the arrival of the direct wave component. 


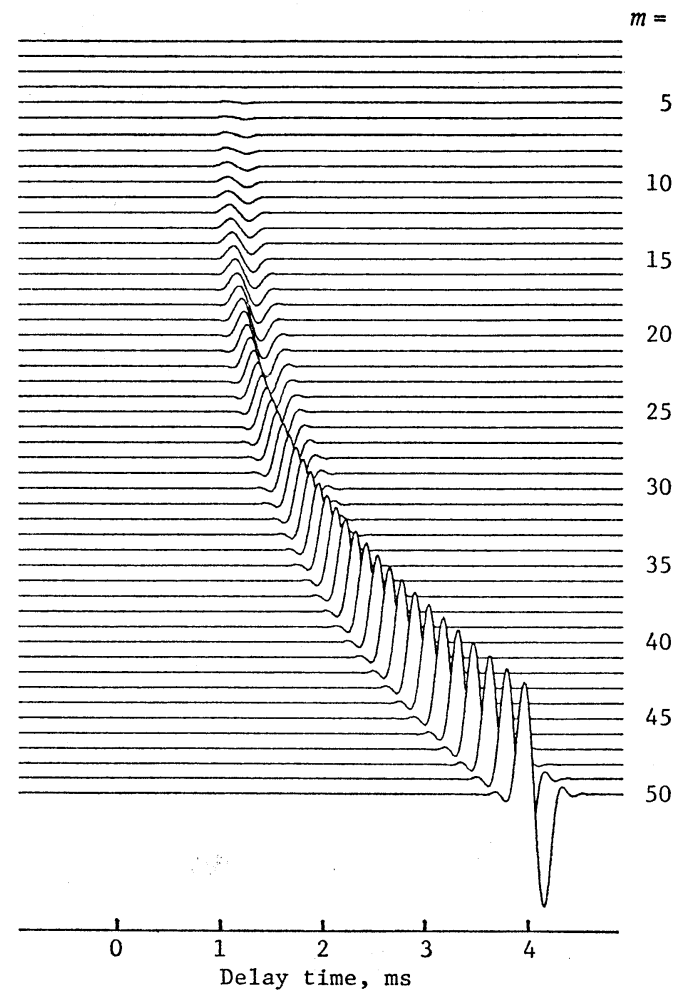

Fig. 5 Wave elements reflected from each oval ring corresponding to the value $m$ in Eq. (4.4) and shown roughly in Fig. 2. Summation of these elements forms the response shown in Fig. 4(a).

impulse response as shown in Fig. 4. The first response in each waveform represents the reflected wave, which may be considered perfectly reflected. The second response is the error caused by the finite upper limit of integration for $a$. In other words, it can be considered the diffracted wave from the periphery of the oval area shown in Fig. 2.

Figure 5 shows the contribution from each oval ring shown roughly in Fig. 2 in the case that $h=$ $140 \mathrm{~cm}$. Each of the wave elements has a differential form of triangular impulse, and summation of these elements forms the impulse response shown in Fig. 4(a).

Figure 6 shows the contribution from each sector shown roughly in Fig. 2. Here amplitudes are enlarged by a factor of 10 . We find that the contribution is largest in the proximity of $\phi=0, \pi$, and that it is unsymmetric with respect to the axis $\phi=\pi / 2$.
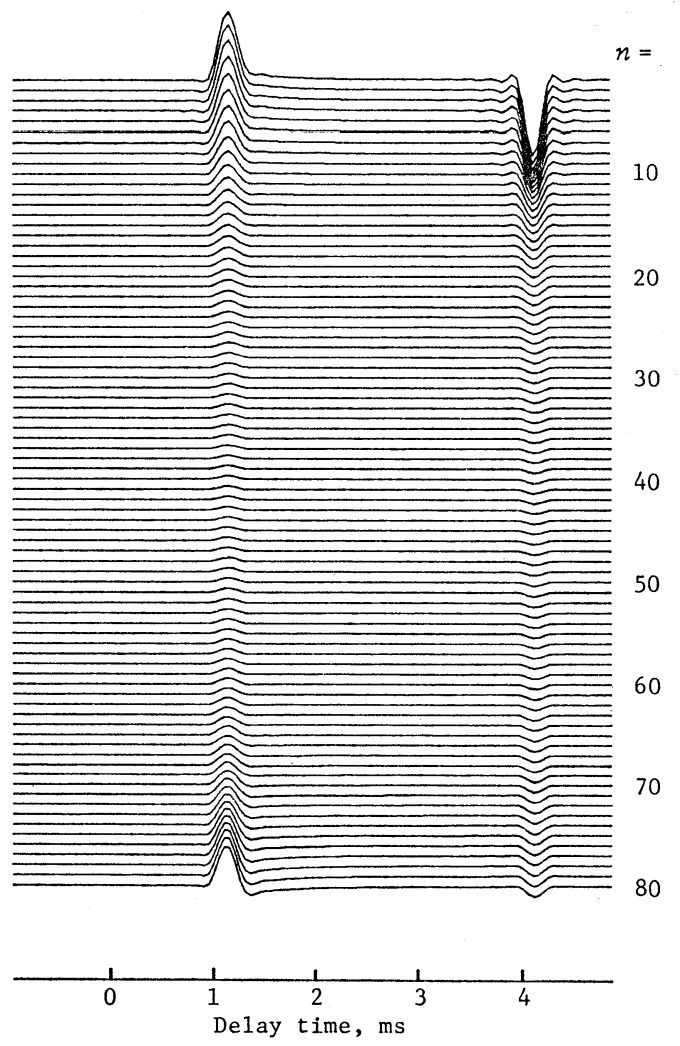

Fig. 6 Wave elements reflected from each sector corresponding to the value $n$ in Eq. (4.5) and shown roughly in Fig. 2.

\subsection{Boundary with Finite Impedance}

We first define the normalized acoustic impedance of the boundary as

$$
\begin{array}{ll}
Z(f)=R(f)+i X(f), \\
R(f)=1+9.08\left(f / \sigma_{R}\right)^{-0.75} & \left(\sigma_{R}=60\right), \\
X(f)=-11.9\left(f / \sigma_{X}\right)^{-0.73} & \left(\sigma_{X}=240\right),
\end{array}
$$

according to Delany and Bazley, ${ }^{9)}$ and the specific admittance as

$$
\nu=1 / Z^{*}(f),
$$

where ${ }^{*}$ denotes complex conjugate. In this model, however, different values of flow resistance $\sigma_{R}$ and $\sigma_{X}$ are used in cgs units. These values have been so chosen that the model is well fit to the experimental results over a turf-covered ground. ${ }^{6)}$ It is strange for a single-layer system that $\sigma_{R} \neq \sigma_{X}$, but it is probable that the turf-covered ground has the property of 


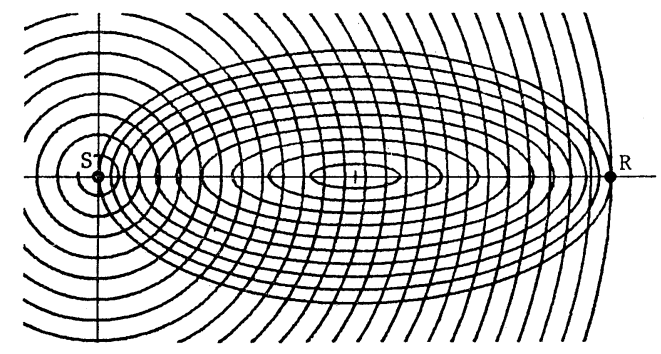

Fig. 7 Concentric circles with center at $\mathrm{S}$; they show the equi-potential curves on the surface.

double-layer system and that the impedance of such a system can be approximated by the model mentioned above.

Next, we must calculate the velocity potential at an arbitrary point $\mathrm{Q}$ on the boundary. It is sufficient to obtain the values $\Psi_{\mathrm{Q}}{ }^{\prime}$ for each concentric circle shown in Fig. 7 using Thomasson's expressions (Eqs. (32) (41) in Ref. 10)) in the case that the receiver is on the boundary. Then the velocity potential at point $\mathrm{R}$ is calculated from Eqs. (3.14) and (3.25). The results are shown in Fig. 8(I). The results calculated from Thomasson's theory only ${ }^{6)}$ are also shown in Fig. 8(II). Both are in complete agreement except for the integration errors mentioned in Sec. 4.1.

When the height $h$ is sufficiently high, the plane wave approximation can be used to obtain $\Psi_{Q}^{\prime}$. Then

$$
\begin{aligned}
& \Psi_{\mathrm{Q}}^{\prime}=\frac{\cos \psi-\nu}{\cos \psi+\nu} \frac{\exp [i k s]}{s}, \\
& \cos \psi=\frac{h}{s} .
\end{aligned}
$$

Figure 9 is the result calculated approximately for $h=140 \mathrm{~cm}$, and it agrees very well with the result shown in Fig. 8(I-a).

\section{SOUND PROPAGATION OVER A BOUNDARY WITH IRREGULARITY}

\subsection{Experimental Results}

Experimental results of sound propagation over a turf-covered ground ${ }^{6)}$ are shown in Fig. 10 for $h=$ 13.3 to $140 \mathrm{~cm}$ with $3.3 \mathrm{~cm}$ step. Here, the irregularities along the broken lines are the components reflected from a tripod used to support a microphone. The others may be considered the effects of irregularities of the boundary. Now pay attention to the concaved portions of the response at $h=140 \mathrm{~cm}$, and trace them upward. Then we obtain three curves (a), (b) and (c) as shown in Fig. 10.
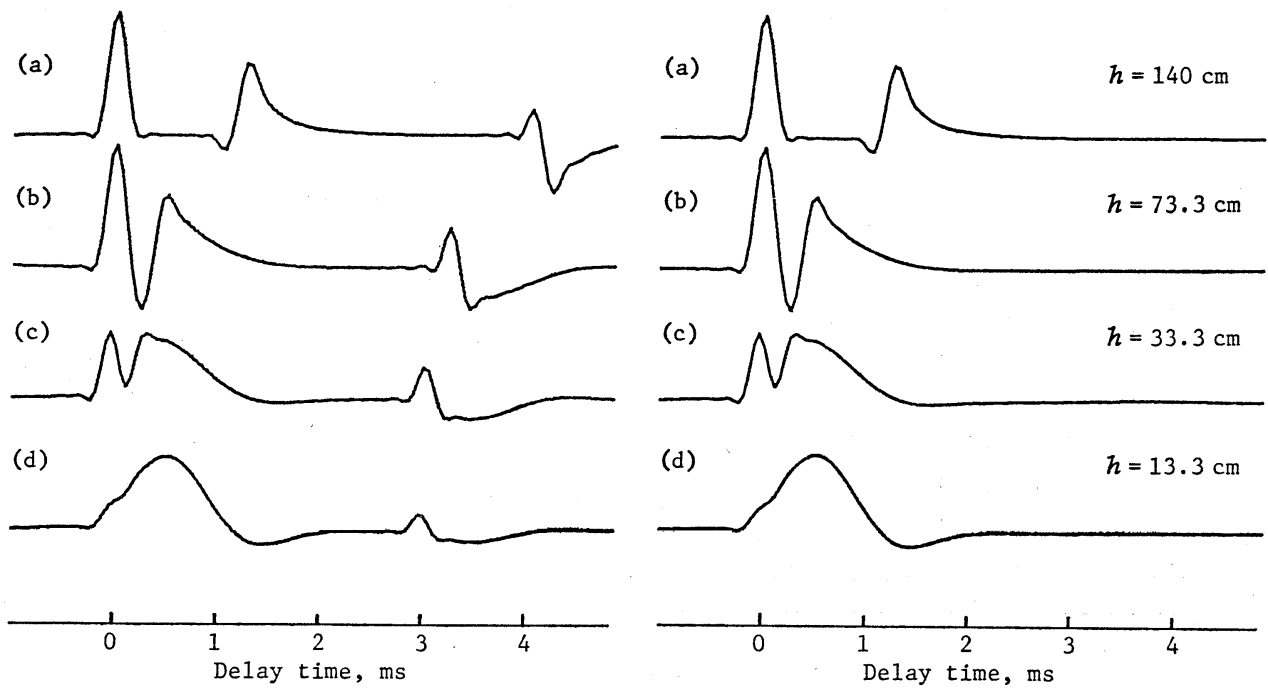

(I)

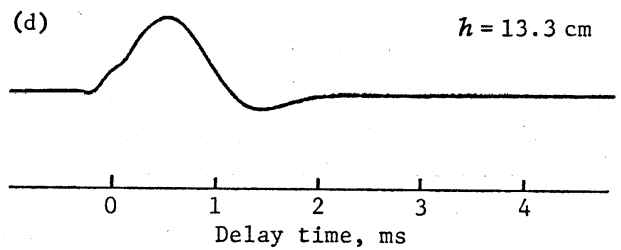

(II)

Fig. 8 (I) Responses of triangular impulse over the boundary of finite impedance with the arrangement of Fig. 2, calculated from Eqs. (3.14), (3.25), (4.8) (4.11) and Thomasson's expressions. ${ }^{10}$

(II) Responses calculated from Eqs. (4.8) (4.11) and Thomasson's expressions only. $\left.{ }^{6}\right)$ 


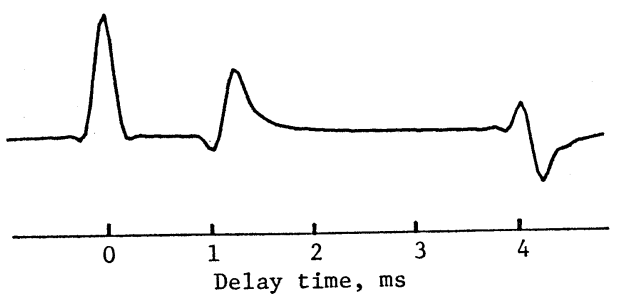

Fig. 9 Response for $h=140 \mathrm{~cm}$ calculated from Eqs. (3.14), (3.25) and (4.8) (4.11) using the approximated potential (Eqs. (4.12) and (4.13)).
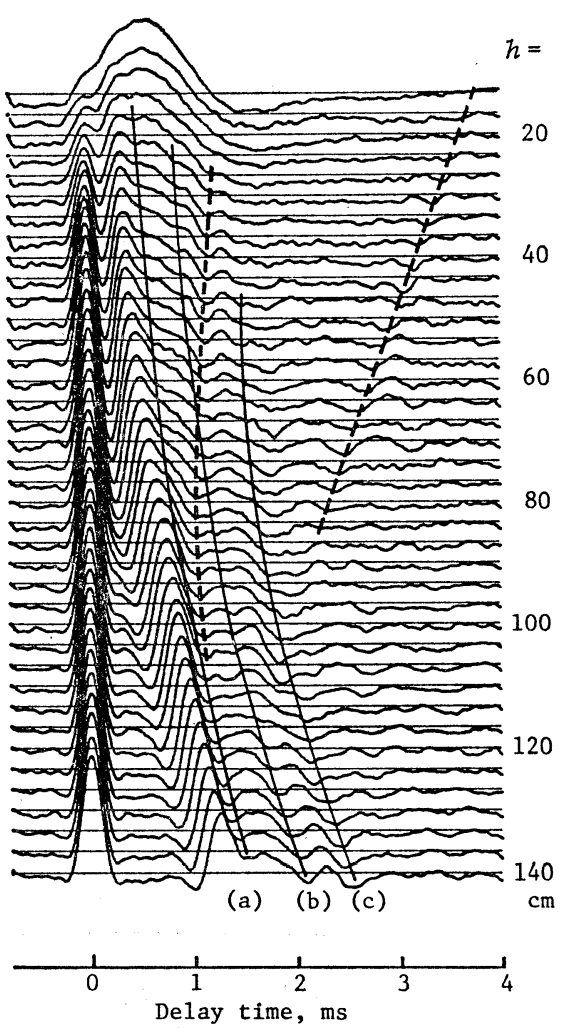

Fig. 10 Experimental results of sound propagation over a turf-covered ground. ${ }^{6)}$

Responses along the broken lines are the reflected components from a tripod used to support a microphone. Concaved portions along the curves (a) $\sim(\mathrm{c})$ are considered the effects of irregularity of the ground surface.
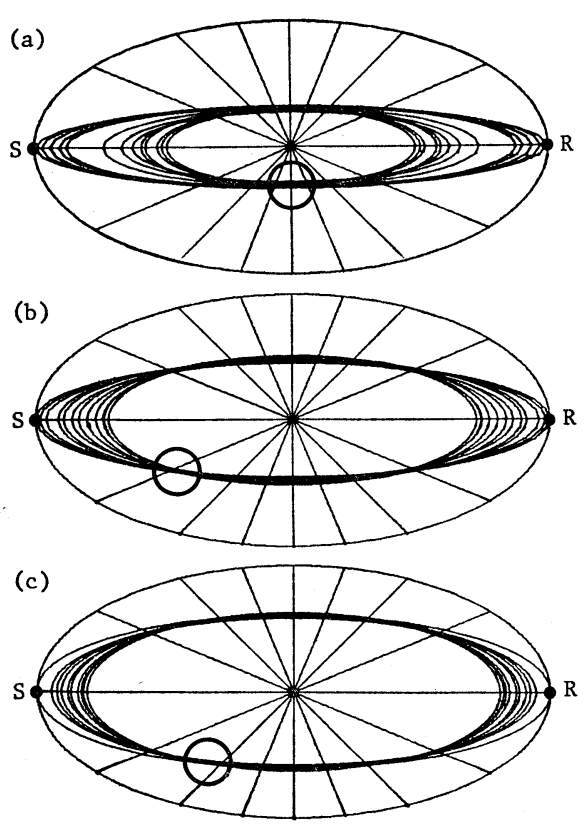

Fig. 11 Estimation of irregular domains from the intersections of ellipses each of which is given by Eqs. (3.22), (3.23) and (5.1) with the values $h$ and $\tau$ corresponding to each concaved portion along the curves (a) (c) shown in Fig. 10.

\subsection{Estimation of Irregular Domains}

Let $\tau$ be the delay time corresponding to each concaved portion. Then the irregularity causing the concave will exist somewhere on the elliptic curve expressed by Eqs. (3.22) and (3.23) for a given $h$ and

$$
a=r_{1}+c \tau,
$$

where $c$ is the speed of sound. Therefore, we can estimate irregular domains from intersections of ellipses corresponding to each concave on each curve (a) $\sim$ (c). The results are shown in Fig. 11 with some ambiguities derived from the symmetrical property of ellipses.

\subsection{Application of the New Theory}

Taking the results obtained in Sec. 5.2 into account, we assume such an irregularity pattern as shown in Fig. 12, although it may not coincide with the actual ground irregularity. We further assume that the directivity of secondary source generated on each area is expressed, instead of Eq. (3.34), as 


\section{Y. MIKI: SOUND PROPAGATION OVER GROUND}

Table 1 Values of the factor $M$ in Eq. (5.2) for each area shown in Fig. 12.

\begin{tabular}{ccccccccccc}
\hline Oval number & $1 \sim 9$ & $10 \sim 17$ & $18 \sim 24$ & 25 & $26 \sim 27$ & 28 & $29 \sim 30$ & 31 & $32 \sim 35$ & $36 \sim 45$ \\
\hline$M$ & 1.0 & 0.6 & 2.0 & 1.0 & 0.8 & 1.0 & 2.0 & 1.0 & 0.1 & 1.0 \\
\hline
\end{tabular}

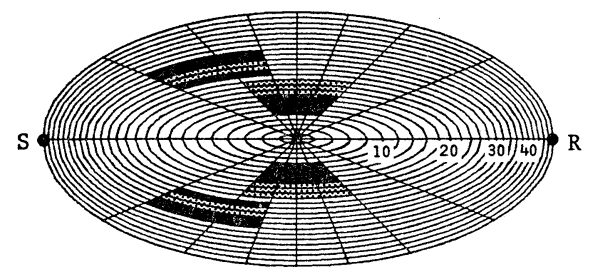

Fig. 12 Assumed irregularity pattern on the ground surface.

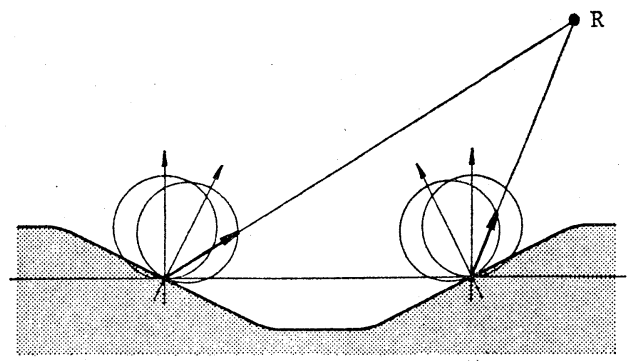

Fig. 13 Change of directivity of the secondary source when the ground surface is locally inclined.

$$
K^{\prime}(\chi)=-\frac{M}{2 \pi}\left(i k-\frac{1}{D}\right) \cos \chi,
$$

where the factor $M$ describes the irregularity of the ground surface and is given as listed in Table 1. This factor represents, for example, the change of directivity when the ground surface is locally inclined as shown in Fig. 13. The degree of inclination, however, is not supposed to be so large as to produce any shadow region or to cause much change in magnitude of the secondary source.

Now we calculate the sound propagation characteristics over the surface with irregularity mentioned above. By using Eqs. (3.25), (4.8) (4.13), (5.2) and Table 1, we obtain the result for $h=140 \mathrm{~cm}$ as shown in Fig. 14. It is in excellent agreement with the measured response shown at the bottom of Fig. 10.

Although the remarkable result is obtained above, it may be difficult to apply the theory to a surface in general, since the analysis is possible only when

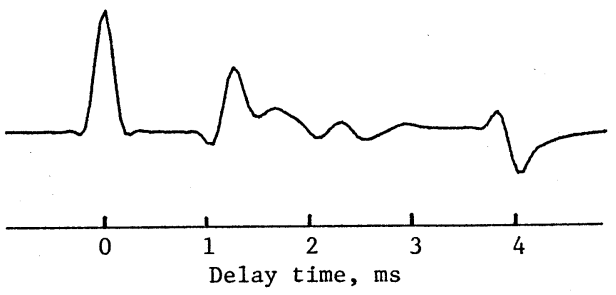

Fig. 14 Response for $h=140 \mathrm{~cm}$ calculated from Eqs. (3.25), (4.8) (4.13) and (5.2) using the irregularity pattern shown in Fig. 12 and Table 1.

both the irregularity pattern and the factor $M$ (or the change of directivity) are perfectly known. The best example of application of this theory may be the case where different kinds of surface of different impedance are distributed with an arbitrary pattern. The procedure for calculation is as follows:

1) In Eq. (3.25), divide the area of integration into sub-areas according to the distribution pattern.

2) For each sub-area, determine the velocity potential $\Psi_{Q}^{\prime}$ using the acoustic impedance of corresponding surface and Thomasson's expressions $^{10)}$ (or Eqs. (4.12) and (4.13)).

3) Calculate Eq. (3.25) using each $\Psi_{Q}^{\prime}$ for each sub-area.

\section{ACKNOWLEDGMENTS}

The author wishes to express his gratitude for the guidance and encouragement received from Prof. Y. Ishii, Institute of Interdisciplinary Research, Faculty of Engineering, University of Tokyo.

\section{REFERENCES}

1) I. Rudnick, "The propagation of an acoustic wave along a boundary," J. Acoust. Soc. Am. 19, 348356 (1947).

2) U. Ingard, "On the reflection of a spherical sound wave from an infinite plane," J. Acoust. Soc. Am. 23, 329-335 (1951).

3) J. A. Stratton, Electromagnetic Theory (McGraw Hill, New York, 1941), p. 573.

4) K. F. Niessen, "Über die entfernten Raumwellen eines vertikalen Dipolsenders oberhalb einer ebenen 
Erde von beliebiger Dielektrizitätskonstante und beliebiger Leitfähigkeit," Ann. Phys. 5, 893-912 (1933).

5) L. Fortuin, "Survey of literature on reflection and scattering of sound waves at the sea surface," J. Acoust. Soc. Am. 47, 1209-1228 (1970).

6) Y. Miki, "Application of the synchronized correlation method to the measurement of sound propagation over a ground surface," J. Acoust. Soc. Jpn. (E) 1, 157-166 (1980).

7) P. M. Morse and K. U. Ingard, Theoretical Acoustics
(McGraw Hill, New York, 1968), p. 266.

8) For example, see H. Yukawa, T. Toyoda, and M. Kawabe, Classical Physics I -Fundamentals of Modern Physics 1, ed. by H. Yukawa (Iwanami, Tokyo, 1975), p. 254 (in Japanese).

9) M. E. Delany and E. N. Bazley, "Acoustical properties of fibrous absorbent materials," Appl. Acoust. 3, 105-116 (1970).

10) S. I. Thomasson, "Reflection of waves from a point source by an impedance boundary," J. Acoust. Soc. Am. 59, 780-785 (1976). 